\title{
Towards an Optimal Observation Strategy for Direct Detection Exoplanet Searches
}

\author{
Jonathan W. Arenberg and Todd E. Schuman \\ Northrop Grumman Space Technology, One Space Park Drive, Redondo Beach, CA, 90278, \\ USA \\ email: jon.arenberg@ngc.com
}

\begin{abstract}
This paper presents a look at ongoing investigations into the strategies needed to characterize the orbits of observed exoplanets in an optimal manner. Also introduced is an efficient manner of the selection of possible orbits consistent with the observation of an exoplanet. Specifically, this work looks at the ability to conclude that a planet's orbit is resident in the habitable zone (HZ) on the basis of two sequential observations. The role of the range of possible semi-major axes is introduced.
\end{abstract}

Keywords. methods: statistical, methods: numerical, methods: data analysis,planetary systems: methods.

\section{Introduction}

This paper is a status report on our investigations regarding the characterization of exoplanetary orbits.

This method is based on a Monte Carlo technique to determine the universe of possible planets consistent with two sequential observations. Eventually, this method will lead to the optimization of the interval between observations. So that the minimum number of observations are required to determine if an orbit is or is not permanently resident in a given zone, typically the so-called habitable zone, where liquid water can exist on the planet's surface.

Our method of investigating the evolution of knowledge of the orbit of a planet is based on sequentially decimating a set that initially represents the universe of possible planets. The universe of possible planets of interest is called $\Omega . \Omega$ is represented as finite set of orbital elements used to calculate the planet's apparent positions on the plane of the sky. For an observation with a given outcome (planet seen or not seen), only those members of $\Omega$ that have the same outcome as the observation are kept. The edited or decimated set is denoted $\Omega_{1}$. The subscript ${ }_{i}$ indicating that all members of $\Omega_{1}$ are consistent with the first observation. The positions of the planets that are members of $\Omega_{1}$ are projected forward in time, at which time a second observation is made and the set is processed by the results of the second observation to yield $\Omega_{2}$ and so on.

To produce the individual members of $\Omega$, denoted $\omega_{i}$, a Monte Carlo method is used to select the various parameters of the orbit, with the orbital inclination, $\theta$, rotation of the orbital plane $\phi$ and true anomaly $\alpha$ are selected uniformly from the interval $[0,2 \pi)$. The eccentricity $\varepsilon$ and semi-major axis a are selected to be uniform for their apastrons and periastrons, which result in a and $\varepsilon$ being distributed in the characteristic camur or bent delta shape. 


\section{Random Orbits Consistent with an Observation, Determination of the $\omega_{i}$}

Simple random generation of numbers to get elements that are consistent with an observation of $\Omega_{1}$ will result in very few close matches to a given observation of a single apparent radial location, resulting in unacceptable computational inefficiency.

The system of equations for $\mathrm{x}, \mathrm{y}$, and $\mathrm{z}$ for an ellipse of semi-major axis a and eccentricity $\varepsilon$ whose orbital plane is inclined to the $\mathrm{x}$-axis by $\theta$ and rotated about the $\mathrm{z}$-axis by $\phi$ is

$$
\begin{gathered}
x(\alpha)=-\sin \theta \cos \phi\left(\frac{a\left(1-\varepsilon^{2}\right)}{1+\varepsilon \cos \alpha} \sin \alpha\right)-\sin \phi\left(\frac{a\left(1-\varepsilon^{2}\right)}{1+\varepsilon \cos \alpha} \cos \alpha\right), \\
y(\alpha)=-\sin \theta \sin \phi\left(\frac{a\left(1-\varepsilon^{2}\right)}{1+\varepsilon \cos \alpha} \sin \alpha\right)+\cos \phi\left(\frac{a\left(1-\varepsilon^{2}\right)}{1+\varepsilon \cos \alpha} \cos \alpha\right), \\
z(\alpha)=\cos \theta\left(\frac{a\left(1-\varepsilon^{2}\right)}{1+\varepsilon \cos \alpha} \sin \alpha\right) .
\end{gathered}
$$

The process used to generate the subset $\Omega_{1}$ for case 2 involves the use of the observed apparent position of the planet, the residency requirement, and Equations (2.1), (2.2), and (2.3). The planet's position is described by five parameters: $\theta, \phi, \alpha$, a, and $\varepsilon$. A single observation does not provide enough information to solve for all five parameters, resulting in an undetermined system. To solve the system, some parameters are chosen randomly within the constraints established by the observation and the residency requirement. The locus of possible values for the remaining variables, which cannot be determined, is a volume in parameter space. It is this space that is sampled via Monte Carlo methods to yield each member of $\Omega_{1}$.

The apparent position of the planet gives a measurement for $\mathrm{y}$ and $\mathrm{z}$. The apparent radial separation $\mathrm{r}_{a p p}$ is given by

$$
r_{a p p}=\sqrt{y^{2}+z^{2}}
$$

The true separation $\rho$ is

$$
\rho=\sqrt{x^{2}+y^{2}+z^{2}} .
$$

For the planet to be of interest, $\rho$ must satisfy the residency requirement and is restricted to the interval [IHZ, OHZ]. Since the magnitude of the third coordinate is still unknown, the true separation must be at least as large as the apparent position, so

$$
\rho \geqslant r_{a p p} .
$$

Combining the condition (2.6) with the residency requirement yields a set of constraints on the random values for $\rho$

$$
\rho \geqslant \max \left(I H Z, r_{a p p}\right)
$$

and

$$
\rho \leqslant O H Z \text {. }
$$

Once $\rho$ is chosen randomly and satisfying (2.7) and (2.8), $\mathrm{x}$ can be calculated using Equations (2.4) and (2.5) to solve for x, resulting in

$$
x= \pm \sqrt{\rho^{2}-r_{a p p}^{2}}
$$

The positive or negative root is chosen randomly to ensure complete sampling of the parameter space. 
Once values have been established for $\{\mathrm{x}, \mathrm{y}, \mathrm{z}\}$, it is possible to generate orbital parameters for orbits that pass through this point. Two additional random variables are selected, $\mathrm{r}_{1}$ and $\mathrm{r}_{2}$ - representing, respectively, the periastron and apastron of the possible planet's orbit. The periastron $r_{1}$ is selected from the interval $[\mathrm{IHZ}, \rho]$ and the apastron $\mathrm{r}_{2}$ from $[\rho, \mathrm{OHZ}]$.

In order to evaluate Equations (2.1), (2.2) and (2.3), values for a and $\varepsilon$ are needed. Expressed in terms of $r_{1}$ and $r_{2}$

$$
a=\frac{1}{2}\left(r_{1}+r_{2}\right)
$$

and

$$
\varepsilon=\frac{r_{1}-r_{2}}{r_{1}+r_{2}}
$$

Substituting Equations (2.10) and (2.11) into Equations (2.1), (2.2), and (2.3) yields a system of three equations and three unknowns (the remaining orbital parameters $\theta, \phi$, and $\alpha$ ). This system is solved numerically for $\theta, \phi$, and $\alpha$. The process is then repeated as many times as desired to create a suitably large Monte Carlo set for $\Omega$ to be used in the analysis of case 2 .

The $r_{1}$ and $r_{2}$ variables are the randomized representations of the $d$ and $D$. That is, we want all orbits that lie between $d$ and $D$, so the nearest point $r_{1}$ is selected to be greater than $\mathrm{d}$ and less than $\mathrm{r}_{2}$ and $\mathrm{r}_{2}$ is less than $\mathrm{D}$.

\section{Evolution of Knowledge of Orbit with Two Observations}

A case where two observations of a planet is considered as an example of this method. Both observations have an apparent position within the HZ, since observation outside would remove a planet from consideration. The question at hand is how likely is the true orbit to lie permanently within the HZ? Is this probability strongly dependent on the universe of possible planets considered. This universe (parent set) can be characterized by the magnitude of the apastron, since all closed obits are considered.

For this current example case, a clone of the sun is the parent star, and for this work the $\mathrm{HZ}$ is on the interval of [0.62 AU,1.52 AU]. It should be noted that the general method works for any interval, as is useful for restricting the description of the orbit of any planet to a specific interval, not just terrestrial like planets.

The initial observation of the planet occurs at an apparent separation of 1 AU. From this apparent position the set $\Omega_{1}$ is generated. The right hand column shows traces of all the orbits consistent with this observation. At time t later, the system is again observed and $\Omega_{1}$ is filtered to give the set of possible orbits consistent with the first and second observations. This set can then be plotted to identify the fraction of possible orbits that are within the habitable zone, those inside the camur delta. The sets $\Omega_{1}$ and $\Omega_{2}$ are calculated for various values of the maximum apastron in the parent set $\Omega$. In this work, the apastrons considered are 2 and 5 AU.

The plot shown in Figure 1 represents the evolution of knowledge of planets orbit based observation. When no observations have been made, the location of the orbit in the $(\mathrm{a}, \varepsilon)$ plane is any closed orbit with an apastron of less than $5 \mathrm{AU}$, this set is represented by the small solid (black) dots. After the first observation, $\Omega_{1}$, is indicated by the small open circles. $\Omega_{1}$ is nearly as widely distributed as $\Omega$ with the exception that some very small a orbits have been ruled out. On the second observation, $\Omega_{2}$ the remaining orbits, show a degree of organization, as indicated by the larger solid (red) dots. All of the orbits with $(\mathrm{a}, \varepsilon)$ consistent with being in the $\mathrm{HZ}$ are the ones inside the region defined by the 


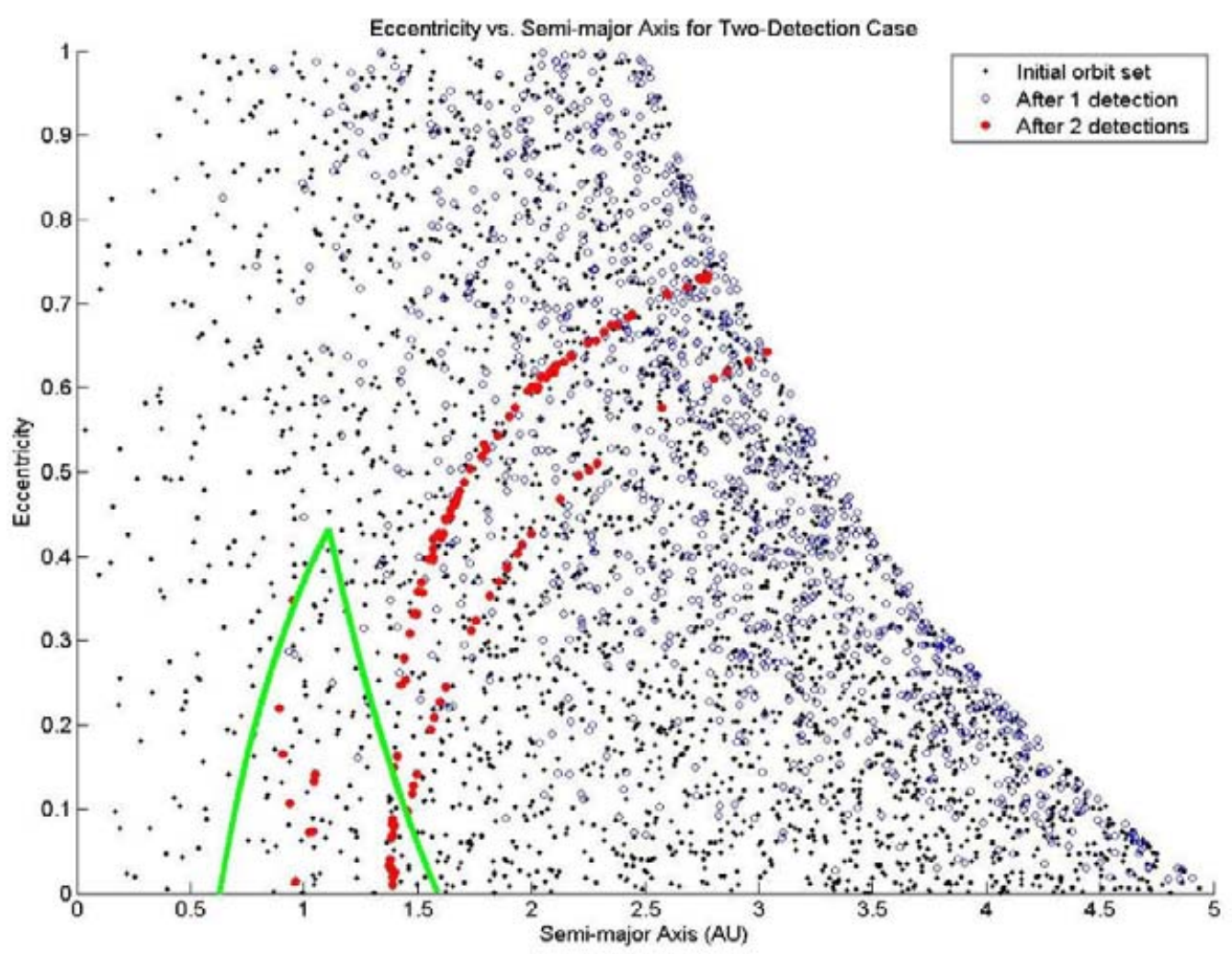

Figure 1. Plot of $\Omega, \Omega_{1}$ and $\Omega_{2}$ in the $(\mathrm{a}, \epsilon)$ plane.

triangle in the lower left quadrant of the plot. Thus, while two observations can tell some data of the planets orbit, it is insufficient to claim residency in the HZ.

Future work is directed at developing algorithms to time the observations to make the determination of in or out of the $\mathrm{HZ}$ as efficient as possible.

\section{Acknowledgements}

This work was performed by Northrop Grumman on internal funds. The authors are grateful for the support. 